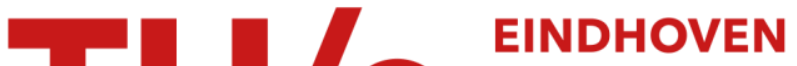 \\ UNIVERSITY OF \\ TECHNOLOGY
}

\section{Conformality of plasma-assisted ALD: physical processes and modeling}

Citation for published version (APA):

Knoops, H. C. M., Langereis, E., Sanden, van de, M. C. M., \& Kessels, W. M. M. (2010). Conformality of plasmaassisted ALD: physical processes and modeling. Journal of the Electrochemical Society, 157(12), G241-G249. https://doi.org/10.1149/1.3491381

DOI:

10.1149/1.3491381

Document status and date:

Published: 01/01/2010

Document Version:

Publisher's PDF, also known as Version of Record (includes final page, issue and volume numbers)

Please check the document version of this publication:

- A submitted manuscript is the version of the article upon submission and before peer-review. There can be important differences between the submitted version and the official published version of record. People interested in the research are advised to contact the author for the final version of the publication, or visit the $\mathrm{DOI}$ to the publisher's website.

- The final author version and the galley proof are versions of the publication after peer review.

- The final published version features the final layout of the paper including the volume, issue and page numbers.

Link to publication

\section{General rights}

Copyright and moral rights for the publications made accessible in the public portal are retained by the authors and/or other copyright owners and it is a condition of accessing publications that users recognise and abide by the legal requirements associated with these rights.

- Users may download and print one copy of any publication from the public portal for the purpose of private study or research.

- You may not further distribute the material or use it for any profit-making activity or commercial gain

- You may freely distribute the URL identifying the publication in the public portal.

If the publication is distributed under the terms of Article 25fa of the Dutch Copyright Act, indicated by the "Taverne" license above, please follow below link for the End User Agreement:

www.tue.nl/taverne

Take down policy

If you believe that this document breaches copyright please contact us at:

openaccess@tue.nl

providing details and we will investigate your claim. 


\title{
Conformality of Plasma-Assisted ALD: Physical Processes and Modeling
}

\author{
H. C. M. Knoops, ${ }^{\text {a,b,*,z }}$ E. Langereis, ${ }^{b, * *}$ M. C. M. van de Sanden, ${ }^{b}$ and \\ W. M. M. Kessels ${ }^{\text {b, } * *}$ \\ ${ }^{a}$ Materials Innovation Institute M2i, 2600 GA Delft, The Netherlands \\ ${ }^{b}$ Eindhoven University of Technology, 5600 MB Eindhoven, The Netherlands
}

\begin{abstract}
For plasma-assisted atomic layer deposition (ALD), reaching conformal deposition in high aspect ratio structures is less straightforward than for thermal ALD due to surface recombination loss of plasma radicals. To obtain a detailed insight into the consequences of this additional radical loss, the physical processes in plasma-assisted ALD affecting conformality were identified and investigated through Monte Carlo simulations. The conformality was dictated by the recombination probability $r$, the reaction probability $s$, and the diffusion rate of particles. When recombination losses play a role, the saturation dose depended strongly on the value of $r$. For the deposition profiles, a minimum at the bottom of trench structures was observed (before reaching saturation), which was more pronounced with larger values of $r$. In turn, three deposition regimes could be identified, i.e., a reaction-limited regime, a diffusion-limited regime, and a new regime that is recombination-limited. For low values of $r$, conformal deposition in high aspect ratio structures can still be achieved, as observed for several metal oxides, even for aspect ratios as large as 30. For high surface recombination loss probabilities, as appears to be the case for many metals, achieving a reasonable conformality becomes challenging, especially for aspect ratios $>10$.
\end{abstract}

(C) 2010 The Electrochemical Society. [DOI: 10.1149/1.3491381] All rights reserved.

Manuscript submitted June 29, 2010; revised manuscript received August 30, 2010. Published September 30, 2010.

Atomic layer deposition (ALD) has become the method of choice for depositing thin films conformally in high aspect ratio (AR) structures. To achieve film conformality, saturation of the surface reactions has to be achieved throughout the three-dimensional (3D) structure. Usually, ALD processes are optimized for planar geometries before deposition on 3D structures is attempted. To achieve conformality in moderate ARs $(A R \leq 10)$, often no or little change in precursor exposure is needed compared to the typical exposure times optimized for the deposition on planar substrates. In this particular case, the ALD process is in the reaction-limited regime. ${ }^{1}$ When going to higher AR structures, both experimental and modeling efforts have shown that a large increase in precursor exposure is required to obtain conformality. The film growth can then be considered to take place in the diffusion-limited regime. ${ }^{1,2}$ Insight into the regime in which the deposition takes place can greatly help in predicting the appropriate process conditions beforehand. For example, it can help to decide whether adjustments to the precursor dosing times are needed and to evaluate whether it is feasible to coat particular 3D structures conformally with acceptable cycle times.

While in thermal ALD, the precursors can generally be considered "stable" in the sense that they remain intact after collisions on already reacted surface sites; this assumption does not hold for all ALD processes. For ozone-based processes, ozone molecules can be lost in surface reactions on many surfaces by forming oxygen molecules, especially at elevated temperatures. ${ }^{3}$ For plasma-assisted ALD processes, the loss of reactive species at surfaces can be even more pronounced, for example, hydrogen radicals recombine easily on metal surfaces. So far, papers on the effects of these loss processes on the dose required for saturation and on the evolution of the deposition profiles in trenches are anecdotal and, to a large extent, the effects itself are unknown.

To address these issues more fundamentally and to obtain a physical model and understanding of the aspects controlling conformality for plasma-assisted ALD, dedicated experimental and theoretical studies are required. Especially, simulations can be a powerful tool because physical effects such as diffusion, surface recombination, and surface reaction can be isolated easily. For example, for chemical vapor deposition, simulations are numerous and have been used to study many aspects of the deposition process including the conformality that can be achieved by various

\footnotetext{
* Electrochemical Society Student Member.

** Electrochemical Society Active Member.

${ }^{\mathrm{z}}$ E-mail: H.C.M.Knoops@tue.nl
}

processes. ${ }^{4,5}$ For thermal ALD, a number of theoretical studies regarding conformality have been reported in the literature. ${ }^{1,2,6-10}$ The two main factors considered in these studies have been the sticking probability $s$ and the AR of the 3D structure. Briefly, for $s=1$ the problem can be solved analytically, ${ }^{2}$ whereas for lower $s$, analytical solutions are more challenging and therefore Monte Carlo simulations are often employed. Two regimes can be identified: the diffusion-limited regime when the value of $s$ is high and the reaction-limited regime when the value of $s$ is relatively low. ${ }^{1}$

In this work, we have developed a Monte Carlo model incorporating surface reaction and surface recombination loss parameters to study the conformality that can be achieved by plasma-assisted ALD processes and to understand how the deposition in 3D structures depends on these parameters. Employing these simulations, the change in dose required for conformal deposition in $3 \mathrm{D}$ structures was investigated as well as the evolution of the deposition profiles and the diffusion of species itself. Besides the two aforementioned regimes that were already observed for thermal ALD, we were able to distinguish a third regime, the so-called recombination-limited regime. The latter regime can account for specific conformality behavior of plasma-assisted ALD in high AR structures.

In the next section, first, the physical processes in plasmaassisted ALD that affect conformality are identified and analyzed. The concepts of surface reaction probability and surface recombination loss probability are discussed. Furthermore, an overview of literature papers on conformal deposition by plasma-assisted ALD is provided as reference information. The combination of these considerations determines the approach and the framework for the Monte Carlo simulations, i.e., the parameters of interest, and the method chosen to investigate the effect of the parameters on the conformality for plasma-assisted ALD.

\section{Plasma-assisted ALD}

Plasma-assisted ALD processes can offer several merits in addition to those already provided by thermal ALD. These merits include a larger temperature window, a higher growth rate, reduced purge times, improved material properties, and an increased choice of precursors and materials. ${ }^{11}$ In plasma-assisted ALD, the reactant step (or reactant pulse) consists of an exposure of the surface to plasma species. These plasma species involve molecules, ions, radicals, electrons, and photons. Of these species, the radicals (reactive atomic and molecular species such as $\mathrm{H}, \mathrm{O}, \mathrm{N}, \mathrm{NH}$, and $\mathrm{NH}_{2}$ ) are considered most important for surface reactions associated with ALD film growth. ${ }^{11}$ Radicals are neutral and therefore unaffected by electric fields that develop in the discharge. Radicals, therefore, dif- 


\begin{tabular}{|c|c|c|c|c|}
\hline Step & Species & $\begin{array}{c}\text { Deposited } \\
\text { material }\end{array}$ & $\begin{array}{l}\text { Reaction } \\
\text { probability } s\end{array}$ & References \\
\hline \multirow{11}{*}{ Precursor } & $\mathrm{Al}\left(\mathrm{CH}_{3}\right)_{3}$ & $\mathrm{Al}_{2} \mathrm{O}_{3}$ & 0.001 & 1 \\
\hline & $\mathrm{Al}\left(\mathrm{CH}_{3}\right)_{3}$ & $\mathrm{Al}_{2} \mathrm{O}_{3}$ & 0.026 & 7 \\
\hline & $\mathrm{Al}\left(\mathrm{CH}_{3}\right)_{3}$ & $\mathrm{Al}_{2} \mathrm{O}_{3}$ & 0.1 & 6 \\
\hline & $\mathrm{Al}\left(\mathrm{CH}_{3}\right)_{3}$ & $\mathrm{Al}_{2} \mathrm{O}_{3}$ & $0.1-0.9$ & 9 \\
\hline & $\mathrm{Hf}(\mathrm{NEtMe})_{4}$ & $\mathrm{HfO}_{2}$ & $0.03-0.6^{\mathrm{a}}$ & 15 \\
\hline & $\mathrm{Ti}\left(\mathrm{NMe}_{2}\right)_{4}$ & $\mathrm{TiO}_{2}$ & $0.02 \pm 0.005$ & 10 \\
\hline & $\mathrm{Ti}\left(\mathrm{O}^{\mathrm{i}} \mathrm{Pr}\right)_{4}$ & $\mathrm{TiO}_{2}$ & $0.04-0.1^{\mathrm{b}}$ & 16 \\
\hline & $\mathrm{Cp}^{*} \mathrm{Ti}(\mathrm{OMe})_{3}$ & $\mathrm{TiO}_{2}$ & 0.01 & 15 \\
\hline & $\mathrm{TiCl}_{4}$ & TiN & $0.006 \pm 0.002$ & 14 \\
\hline & $\mathrm{ZnEt}_{2}$ & $\mathrm{ZnO}$ & 0.007 & 1 \\
\hline & $\mathrm{SiCl}_{4}$ & $\mathrm{SiO}_{2}$ & $10^{-8}$ & 1 \\
\hline \multirow[t]{2}{*}{ Reactant } & $\mathrm{H}_{2} \mathrm{O}$ & $\mathrm{Al}_{2} \mathrm{O}_{3}$ & $0.01-0.1$ & 9 \\
\hline & $\mathrm{O}_{3}$ & $\mathrm{Al}_{2} \mathrm{O}_{3}$ & $0.001-0.01$ & 9 \\
\hline \multirow[t]{3}{*}{ Plasma } & $\mathrm{O}$ & $\mathrm{Al}_{2} \mathrm{O}_{3}$ & $0.1-0.9$ & 9 \\
\hline & $\mathrm{H}$ & TiN & $0.0003 \pm 0.0001$ & 14 \\
\hline & $\mathrm{N}$ & TiN & $0.01 \pm 0.002$ & 14 \\
\hline
\end{tabular}

${ }^{\text {a }}$ Corresponding to the substrate temperature range of $180-270^{\circ} \mathrm{C}$.

${ }^{\mathrm{b}}$ Corresponding to the substrate temperature range of $125-225^{\circ} \mathrm{C}$.

fuse similarly as the reactants used in thermal ALD. Although radicals are considered most important, other plasma species can also have an influence on the growth process. Charged particles (ions and electrons) are influenced by electric fields and can consequently cause additional (detrimental or beneficial) effects such as surface ion bombardment. Furthermore, (vacuum)ultraviolet [(V)UV] photons generated in the plasma and on surfaces can also have an influence (e.g., defect creation at $\mathrm{Si}_{-} \mathrm{SiO}_{2}$ interfaces). ${ }^{12}$ With this in mind, plasma-assisted ALD processes can be divided into three configurations: ${ }^{11}(i)$ direct plasma ALD, where the sample is part of the plasma generation zone such that it can receive a considerable ion and photon flux; (ii) remote plasma ALD, where the plasma is produced upstream but with the plasma species still present above the substrate with lower energies and potentially smaller fluxes; and (iii) radical-enhanced ALD, where the plasma is generated at a large distance from the sample such that hardly any charged particles or photons reach the substrate. In most cases, effects of ion bombardment and (V)UV photon flux are relatively small and can therefore be neglected to give a fair approximation. This can even hold for direct plasma ALD, e.g., when operating the plasma at higher pressures.

Reaction probability.- The ALD reactions involving the precursor and reactive surfaces during the precursor pulse, e.g., ligand exchange, dissociation, association, etc., ${ }^{13}$ are often generalized by a reaction probability per collision, i.e., a sticking probability $s$. Similarly, also the ALD reactions during the reactant step can be generalized by a reaction probability per collision $s$. For plasma radicals, these reactions in the ALD growth process can be (i) oxidation: oxygen radicals can oxidize organic ligands and the metal atoms deposited; (ii) abstraction: hydrogen radicals can, for instance, react with halide surface groups in abstraction reactions; (iii) reduction: hydrogen radicals can act as a reducing agent for metal atoms; and (iv) nitridation: nitrogen radicals can be incorporated into the material. A wide range of reaction probabilities $s$ can be found in the literature, as shown in Table I. Sometimes, even a wide range of $s$ values are reported for the same process, e.g., $\mathrm{Al}\left(\mathrm{CH}_{3}\right)_{3}$ adsorption. This can, in part, be explained by the wide range of methods used to determine the probabilities, ranging from ab initio density functional theory calculations ${ }^{9}$ to direct beam experiments, ${ }^{14}$ but also by varia-

\begin{tabular}{|c|c|c|c|}
\hline Radical & Surface & $\begin{array}{l}\text { Recombination } \\
\text { probability } r\end{array}$ & Reference \\
\hline \multirow{12}{*}{$\mathrm{H}$} & Silica & $0.00004 \pm 0.00003$ & 42 \\
\hline & Alumina & $0.0018 \pm 0.0003$ & 43 \\
\hline & Pyrex & $0.0058 \pm 0.0018$ & 20 \\
\hline & Stainless steel & $0.032 \pm 0.015$ & 43 \\
\hline & Silicon & $0.70 \pm 0.10$ & 44 and 45 \\
\hline & Titanium & 0.35 & 20 \\
\hline & Aluminum & 0.29 & 20 \\
\hline & Nickel & $0.20 \pm 0.09$ & 43 \\
\hline & Copper & 0.14 & 20 \\
\hline & Gold & $0.15 \pm 0.05$ & 20 \\
\hline & Palladium & $0.07 \pm 0.015$ & 20 \\
\hline & Platinum & 0.03 & 20 \\
\hline \multirow[t]{4}{*}{$\mathrm{N}$} & Silica & $0.0003 \pm 0.0002$ & 42 \\
\hline & Stainless steel & 0.0063 & 46 \\
\hline & Silicon & 0.0016 & 46 \\
\hline & Aluminum & 0.0018 & 46 \\
\hline \multirow[t]{9}{*}{$\mathrm{O}$} & Silica & $0.0002 \pm 0.0001$ & 42 \\
\hline & Pyrex & 0.000045 & 18 \\
\hline & Aluminum oxide & 0.0021 & 18 \\
\hline & Zinc oxide & 0.00044 & 18 \\
\hline & Ferric oxide & 0.0052 & 18 \\
\hline & Cobalt oxide & 0.0049 & 18 \\
\hline & Nickel oxide & 0.0089 & 18 \\
\hline & Cupric oxide & 0.043 & 18 \\
\hline & Stainless steel & $0.070 \pm 0.009$ & 47 \\
\hline
\end{tabular}

tions in experimental and theoretical accuracies. Temperaturedependent values of $s$ have also been reported; for example, an increase in $s$ with temperature was found for $\mathrm{Hf}(\mathrm{NEtMe})_{4}$ and $\mathrm{Ti}\left(\mathrm{O}^{\mathrm{i}} \mathrm{Pr}\right)_{4}$ precursor adsorption. ${ }^{15,16}$ Regarding the reactant step in plasma-assisted ALD, oxygen radicals appear the most reactive, whereas hydrogen radicals have generally the lowest reactivity. Commonly, reaction probabilities fall in the range of 0.1-0.001.

Recombination loss probability. - In the case of plasma-assisted ALD, the reactants can also be lost in recombination processes at the surfaces, this in addition to the loss by ALD surfaces reactions. For instance, a hydrogen radical can recombine with a hydrogen atom on the surface to form molecular hydrogen. This hydrogen molecule has no further influence on the ALD surface reactions and becomes again a part of the hydrogen source gas of the plasma. The surface recombination probability depends on the type of radical and on the material of the surface with which the radical collides. Table II lists literature values for these surface recombination loss probabilities $r$ for hydrogen, nitrogen, and oxygen radicals on a set of materials. A large range of loss probabilities is reported. In general, recombination on oxides is low, whereas metals show higher $r$ values. An inverse relation between hydrogen surface radical recombination probability and the electron work function for some materials has been reported. ${ }^{17}$ Overall agreement with this trend is found for the part of Table II starting at silicon (low work function, high recombination) and ending at platinum (high work function, low recombination). In general, nitrogen and oxygen radicals have lower loss probabilities than hydrogen radicals. Oxygen radicals are suggested to have higher recombination probabilities on oxide surfaces with atoms containing incomplete $\mathrm{d}$ shells $(\mathrm{Mn}, \mathrm{Fe}, \mathrm{Co}, \mathrm{Ni}$, and $\mathrm{Cu}){ }^{18}$ Furthermore, significant temperature and pressure dependencies can exist. For instance, Greaves and Linnett reported an increase in the loss probability for oxygen on silica of 2 orders of magnitude from $1.6 \times 10^{-4}$ to $1.4 \times 10^{-2}$ for temperatures ranging from 20 to $600^{\circ} \mathrm{C}^{19}$ Wood and Wise reported that most metals have a fairly 
constant recombination probability for hydrogen radicals over a wide temperature range. ${ }^{20}$ The surface conditions during ALD can differ from those in the reported studies, affecting the value of $r$.

Conformal films deposited by plasma-assisted ALD.- Despite the possibility of recombination of radicals at surfaces, numerous studies have shown that fair to high conformality can be reached in high AR structures by plasma-assisted ALD. For instance, for ALD of metal oxides with $\mathrm{O}_{2}$ plasmas, $\sim 100 \%$ conformality has been reached for $\mathrm{Al}_{2} \mathrm{O}_{3}$ in pores with $\mathrm{AR}=8,{ }^{21}$ for $\mathrm{TiO}_{2}$ deposition in trenches with $\mathrm{AR}=9,{ }^{16,22}$ and for $\mathrm{Ta}_{2} \mathrm{O}_{5}$ in trenches with $\mathrm{AR}$ $=11 .^{23}$ Regarding $\mathrm{H}_{2}$ plasmas used for ALD of metals and metal nitrides, $>95 \%$ step coverage for $\mathrm{TiN}$ in pores with $\mathrm{AR}=10$ has been reported; ${ }^{24} \sim 100 \%$ for TaN in pores with $\mathrm{AR}=10,{ }^{25} \sim 100 \%$ conformality for $\mathrm{Cu}$ in trenches with $\mathrm{AR}=9,{ }^{26}$ as well as conformal growth for $\mathrm{Ag}$ in trenches with $\mathrm{AR}=9^{27}$ were also reported. For ALD of Ta with a $\mathrm{H}_{2}$ plasma, $\sim 100 \%$ conformality was found up to trenches with $\mathrm{AR}=15$, whereas the rest of the trench $(\mathrm{AR}=40)$ had a conformality of $40 \% .{ }^{28}$ Deposition profile information such as this is rare because conformality can be difficult to assess and requires transmission electron microscopy or scanning electron microscopy analyses with relatively thick films. However, this kind of profile information is crucial to understand the evolution of the deposition profile within the high AR structure during the ALD process. The lack of papers on conformal growth in higher ARs could indicate those being more difficult to coat, albeit that this has not been stated explicitly in the literature.

Modeling approach.- The goal of this work is to obtain a detailed understanding of the mechanisms determining the conformality that can be reached by plasma-assisted ALD. To this end, we have developed a Monte Carlo model to simulate the basic and relevant aspects of the plasma-assisted ALD process, which have been outlined in the previous section. With these simulations, a detailed insight into the effects of reaction probability, loss probability, and AR on conformality is obtained. The main simulation parameters that are discussed are the dose required to achieve saturation in 3D structures, the deposition profile in these structures before saturation has been reached, and the profile of the wall collisions of the reactive species. Combining the results offers an insight into the ability of plasma-assisted ALD to deposit conformal films in 3D structures.

\section{Monte Carlo Simulations}

In this section, first, the simulation and the simulated parameters of interest are defined. Our Monte Carlo model is similar to those reported earlier for thermal ${ }^{1,6-10}$ and plasma-assisted ALD. ${ }^{29,30}$ For simplicity and fast computation, we have chosen a two-dimensional (2D) Monte Carlo simulation. Only one ALD half-reaction is considered at a time, assuming that the previous half-reaction was fully saturated. A possible overlap between the precursor and reactant pulse is not investigated as these can be assumed to be wellseparated by purge steps. Particles that represent precursor molecules or radicals are simulated one at a time, which is valid because the particles only interact with the walls. Due to the small feature size with respect to the mean free path (high Knudsen number), the flow is molecular and particle-particle interaction can be neglected. The various ALD surface reactions (Table I) are generalized with a single reaction probability $s$. The reaction probability $s$ can be viewed as a generalized sticking probability, defined as the probability of a surface reaction resulting in a change of the surface contributing to the ALD process. With respect to $s$, both the precursor and the reactant half-cycle are equivalent in behavior. Based on the values in Table I, $s$ is chosen in the range $0.001-1$ for the simulations. We also generalized the surface recombination loss processes (Table II) by a single loss probability $r$. This generalized process can be defined as a surface reaction where the incoming reactant species
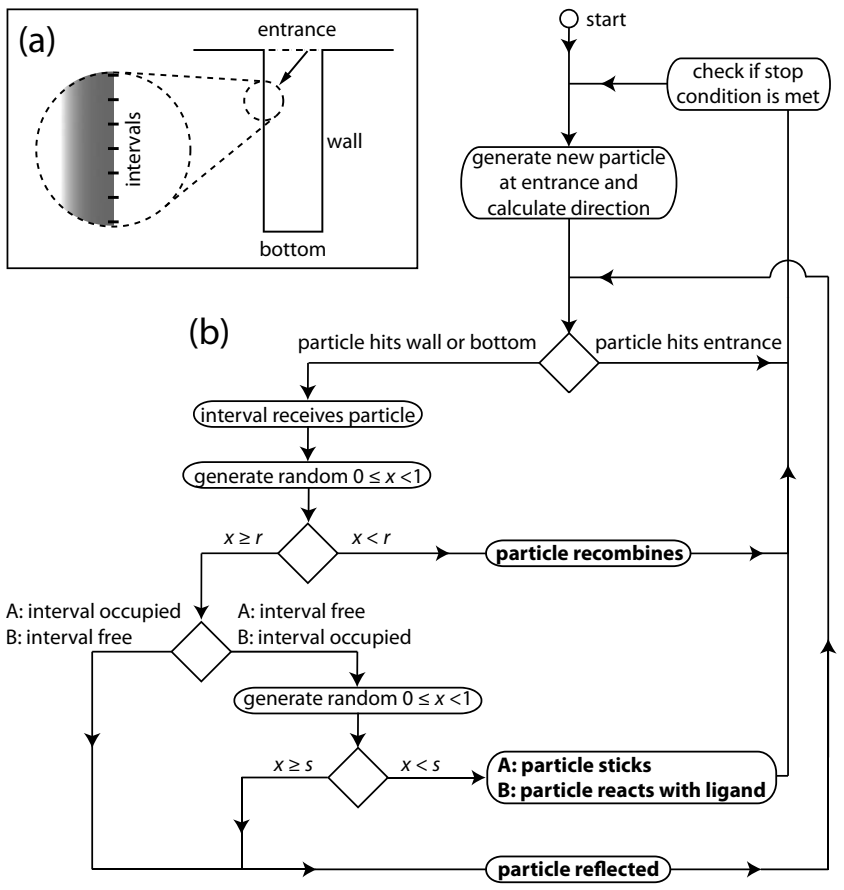

Figure 1. (a) Schematic of the simulation domain, i.e., a $2 \mathrm{D}$ trench of specific AR. Particles (represented by an arrow) are generated at the entrance of the trench. The inset shows the representation of the discrete surface intervals. (b) Flowchart of the Monte Carlo simulations for the ALD halfreactions. After collision with the sidewalls or bottom of the trench, a particle either recombines (depending on $r$ ), reacts (depending on $s$ and the state of the interval), or reflects. Particles are generated until a stop condition is met.

changes such that it cannot contribute anymore to the ALD process. Based on Table II, $r$ is chosen within the range $0-0.9$ for the simulations.

The simulation domain is a $2 \mathrm{D}$ trench with specific AR (ratio between depth and width) consisting of a trench entrance at the top, a trench sidewall on each side, and a trench bottom (Fig. 1a). The trench sidewalls and the trench bottom represent the surfaces for growth and are divided into discrete intervals (inset Fig. 1a). The simulation proceeds as illustrated in Fig. 1b: A particle is emitted with a cosine-distributed random direction at a random location at the entrance of the trench. ${ }^{5}$ The random location at the entrance is also cosine-distributed. Subsequently, the intersection of the trajectory of the particle with the trench surface is calculated. At the corresponding surface interval, one out of three processes can occur: (i) The particle can undergo surface recombination with a loss probability $r$; (ii) the particle can react and contribute to the ALD process with a reaction probability $s$; and (iii) the particle can be reflected, assuming that the particle is emitted with a new random direction according to a cosine distribution. ${ }^{5}$ For the reflected particle, the algorithm starts over, beginning with the calculation of the new trajectory and intersection point. If the new intersection point is at the entrance of the trench, the particle is considered lost and a new particle is generated (unless the stop condition has been met). Otherwise, one of the three processes (recombination, reaction, or reflection) occurs again. Depending on whether ALD half-cycle A (precursor pulse) or B (reactant pulse) is meant to be simulated, a surface interval on which a reaction can occur can be interpreted as "empty" (case A) or "occupied" (case B). The ALD reaction then either "occupies the empties interval" (case A) or "empty the occupied interval" (case B). Simulationwise, cases A and B are equivalent, and therefore, results intended for case A also hold for case B if they have the same values for $s$ and $r$.

The simulation program was written in $\mathrm{C}++$ code and the program is run on a desktop computer where an average simulation of 


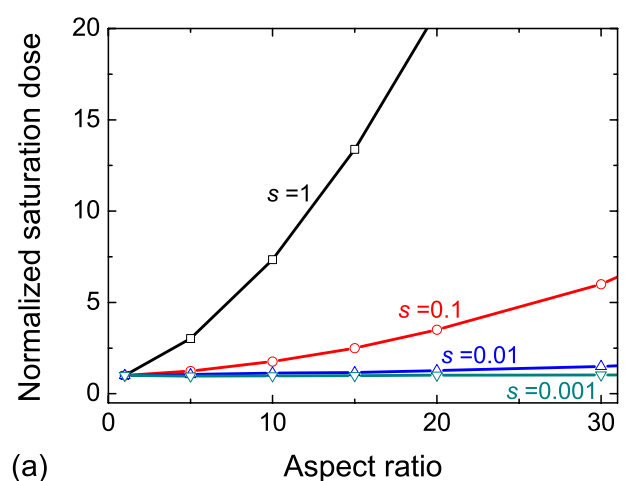

(a)

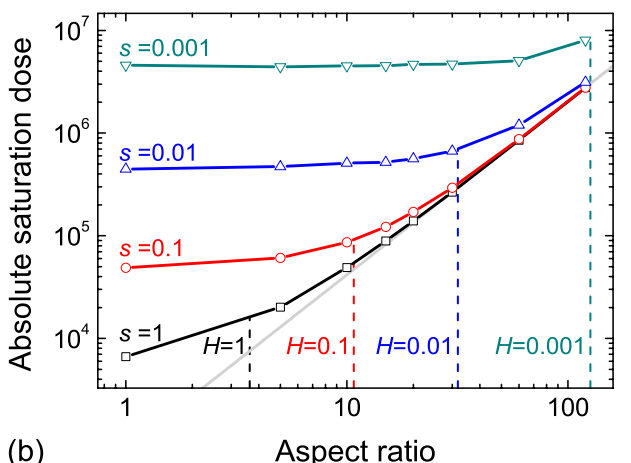

(b)

Aspect ratio
Figure 2. (Color online) (a) Normalized saturation dose as a function of AR for reaction probabilities $s$ ranging from 0.001 to 1 . The dose is normalized to the dose required for an AR of 1. (b) Absolute saturation dose as a function of AR for reaction probabilities $s$ ranging from 0.001 to 1. For (a) and (b), recombination loss is not included, i.e., $r=0$. Dashed vertical lines indicate the AR where the hopping coefficient $H$ is equal to the reaction probabilities $s$ used in the simulations. a trench with $\mathrm{AR}=20(20,000 \times 1000$ intervals and $s=0.01, r$ $=0.1)$ took $30 \mathrm{~s}\left(1.3 \times 10^{7}\right.$ particles) to reach (nearly full) saturation. The profiles considered are the average of five simulations and are smoothed over 1000 intervals to reduce statistical noise.

It is important to stress the simplicity of the model used for the Monte Carlo simulations. A change in the AR during the growth process is not considered, which is valid when the thickness of the deposited film is much smaller than the width of the trench. Furthermore, the influence of the particle velocity and gas and substrate temperature dependence is not considered explicitly. The influence of these parameters can be studied through their influence on the values of $s$ and $r$. Regarding the surface intervals, one reaction changes a surface interval into either occupied or empty, corresponding to one single ligand occupying a surface interval and only one radical being sufficient to empty the surface interval. No chemistry other than the generalized ALD reaction and recombination processes is considered. The simulation domain is fully $2 \mathrm{D}$, corresponding to a quasi-3D trench. Furthermore, the reaction and recombination loss processes are simulated in a very general way by a constant reaction and loss probability. In reality, both the reaction and loss probabilities can depend on the state of the surface. For example, a surface with unreacted ligands can have a different recombination loss probability than a surface without ligands.

Mainly, three types of information were extracted from the simulations; their definitions and interpretations are $(i)$ the dose: The number of particles generated can be considered as a representation of the applied dose. When the dose equals the dose required to achieve saturation of the 2D trench surface, the dose is called "saturation dose"; (ii) the deposition profile: The deposition profile along the trench wall can be interpreted as a thickness profile in case multiple cycles would be used, i.e., representing the thickness as a function of depth. Another interpretation would be the occupation of reactive surface sites along the trench wall after a single half-cycle; and (iii) the wall collisions profile: The profile of the number of collisions made by the particles along the trench wall. The wall collisions profile provides information on the particle distribution and on particle diffusion within the trench.

Results were extracted from the simulations by defining specific conditions for ending the simulations. These stop conditions are related to the physical effect investigated: $(i)$ The $99 \%$ saturation case, which represents practically the fully saturated situation in which the deposition profile itself provides no physical information but the required dose does, because it represents the saturation dose; (ii) the $90 \%$ saturation case in which the simulation is terminated when $90 \%$ of the intervals have reacted. This situation gives an insight into the gradual change of the deposition profile during exposure. The unsaturated deposition profile can, for instance, identify bottleneck regions where achieving growth is more difficult; and (iii) the case in which a preset number of particles are generated, which can be used to compare two situations with the same dose or to consider the deposition profile after a fraction of the saturation dose.

\section{Results and Discussion}

The conformality of plasma-assisted ALD is investigated in the following sections using the aforementioned stop conditions. In the first part, the surface recombination loss of radicals is not included in the simulations $(r=0)$ to simulate the case for plasma-assisted ALD with no recombination. This case also corresponds to the thermal ALD case and serves, furthermore, as a reference for the case with recombination. Subsequently, surface recombination is included $(r>0)$ and its effects on the saturation dose and on the deposition profile are studied. Particle diffusion is studied by considering the wall collisions profiles for the three deposition regimes. The behavior of these regimes is addressed and the regimes are demarcated using transition conditions. Finally, the implications for plasma-assisted ALD are discussed and the applicability to other processes with the possibility of surface loss (e.g., ozone-based processes) is addressed.

No surface recombination loss $(r=0)$. - First, the case without surface recombination loss is considered $(r=0)$ to obtain a basic understanding of ALD in 3D structures and to investigate the conformality for plasma-assisted ALD in this specific case. Furthermore, it provides the opportunity to test the model against general observations in thermal ALD experiments and simulations. Figure 2a shows the normalized saturation dose as a function of AR for various reaction probabilities $s$. The dose has been normalized by dividing it by the dose required for an AR of 1 (close to planar geometry). Because ALD processes are usually optimized for planar substrates, this normalization directly provides a measure of the extension of the dose needed to coat 3D structures conformally. For instance, if a $2 \mathrm{~s}$ dose is required for a planar substrate, a normalized dose of 5 would represent a necessary extension of the dose to $10 \mathrm{~s}$. For $s=1$, a large increase in normalized saturation dose is observed, similar to the trend predicted by Gordon et al. ${ }^{2}$ For lower values of $s$, the normalized saturation dose shows much less dependence on the AR. In Fig. 2b, the saturation dose is replotted as a function of AR but now without normalization, i.e., the dose represents the total number of generated particles. For the near planar case, this absolute saturation dose is inversely proportional to $s$, which is expected because the same holds for planar substrates. For high ARs, the trends start to overlap with the one for the $s=1$ case. The latter explains the success of the model reported by Gordon et al. (which assumes $s=1$ ) in predicting the dose in the case of high ARs. Consequently, although experimental data might agree with the dose predicted by the model of Gordon et al., this agreement does not mean that $s$ is close to 1 because $s$ values ranging from 0.01 to 1 all require the same amount of dose for very high ARs (e.g., AR $=100$ ).

From the results in Fig. 2, two regimes can be identified. One where the saturation dose varies little with the AR and depends only on the reaction probability $s$ (reaction-limited), and the other where 


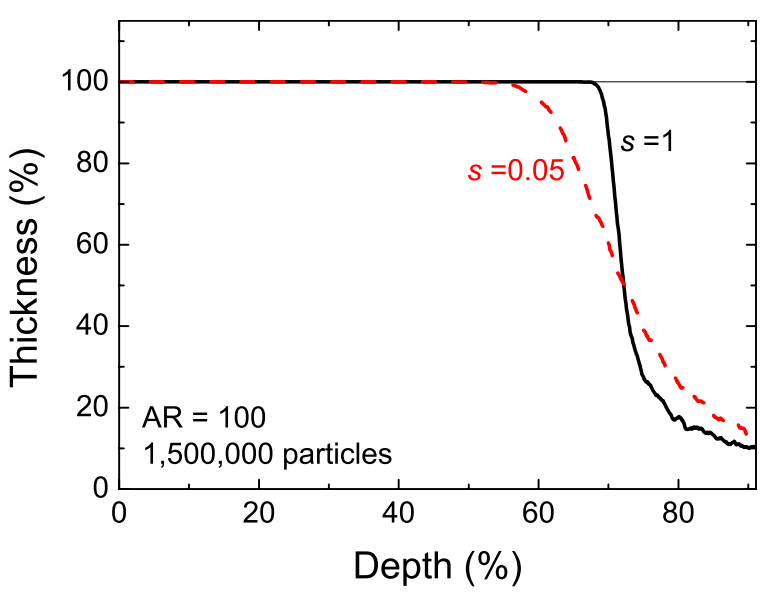

Figure 3. (Color online) Thickness as a function of depth for a trench with $\mathrm{AR}=100$ for simulations with reaction probability $s=1$ and 0.05 ; in both cases $1.5 \times 10^{6}$ particles were generated. Recombination loss is not included $(r=0)$.

the dose increases strongly with AR but depends little on the reaction probability $s$ (diffusion-limited). Elam et al. reported the same two regimes and suggested a "hopping coefficient" $H$ to determine in which regime the process takes place. ${ }^{1} H$ is the average probability that the interval reached by a particle is empty and can be determined from a one-dimensional random walk analysis. $H$ has been shown to be inverse quadratically related to the AR $\left(H=16 / \mathrm{AR}^{2}\right)$, indicating that it becomes progressively more difficult for the particles to reach an empty site for higher ARs. Using $H$, the transition between the regimes can be defined. In the case $s \ll H$, the process is reaction-limited, whereas for $s \gg H$, the process is in the diffusion-limited regime. ${ }^{1}$ Figure $2 \mathrm{~b}$ includes vertical dashed lines to indicate for each simulated value of $s$ the AR at which $s$ equals $H$. The positions of these vertical lines agree reasonably well with the points at which the lines approach the tangent of the $s=1$ case, thereby marking the transition between regimes for each value of $s$. Apparently, although the relation $H=16 / \mathrm{AR}^{2}$ was derived for pores, the condition $s=H$ can also serve as a measure for the transition between reaction- and diffusion-limited regimes for the trench structures, which are used in our simulations. Interestingly, many processes listed in Table I $(s \leq 0.01)$ would be in the reactionlimited regime for the ARs in Fig. $2 \mathrm{a}(\mathrm{AR} \leq 30)$. Rose et al. argued that a high value for $s$ is desired in all cases for ALD. ${ }^{15} s$ is only of influence on the saturation dose for the reaction-limited regime. This means that for very high AR structures, the situation for which ALD is particularly powerful, increasing the value of $s$ yields no advantage because the process is in the diffusion-limited regime.

Because in the diffusion-limited regime the saturation dose for different $s$ values is the same, it is interesting to compare the deposition profiles when the deposition profiles are not fully saturated. Figure 3 shows a deposition profile for a high AR of 100 for $s$ $=0.05$ and $s=1$, both with $1.5 \times 10^{6}$ particles generated. As expected from Fig. $2 b$, the same amount of dose results in nearly the same depth of the trench that is coated conformally. One clear difference, however, is the slope of the deposition front (i.e., the region where the thickness drops). Similar to Rose and Bartha, we observe a lower slope of the deposition front for a lower value of $s .^{10}$ However, the slope at the deposition front cannot always be attributed to the value of $s$. For instance, Perez et al. also observed a particular slope at the deposition front, but in this case, the slope was caused by the reduction of the AR during growth because the film thickness was of the same order of magnitude as the width of the 3D structure. $^{31}$

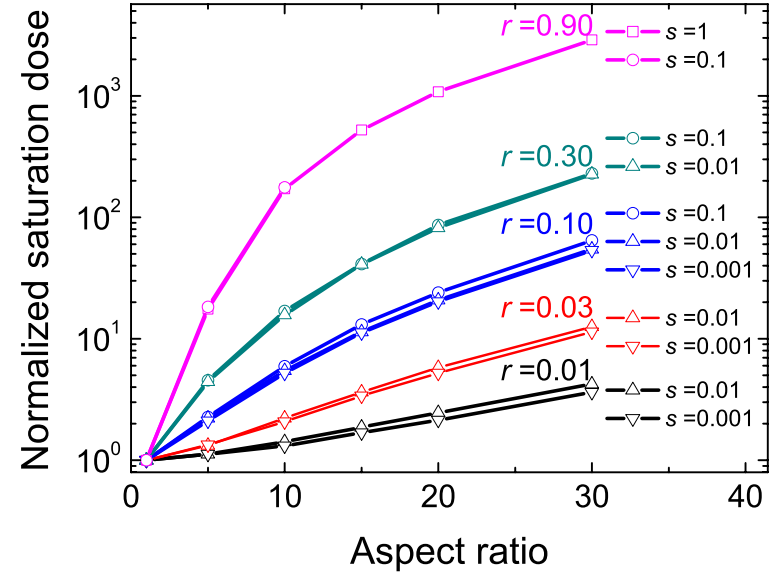

Figure 4. (Color online) Normalized saturation dose as a function of AR for various recombination loss probabilities $r$ and reaction probabilities $s$. The dose is normalized to the dose required for an $\mathrm{AR}$ of 1 . The trends with the same $r$ value but different $s$ values overlap.
Nonzero surface recombination loss $(r>0)$. - The possibility of recombination loss forms a marked difference between plasmaassisted and thermal ALD. By comparing simulation results with zero and with nonzero recombination loss probability $r$, the effects of the recombination loss can be studied. Figure 4 shows the normalized saturation dose as a function of the AR for various loss probabilities $(r=0.01-0.9)$. For each loss probability, a selection of reaction probabilities (ranging from $s=0.001$ to 1 ) was simulated. For each value of $r$, the range of $s$ values selected has little effect on the required normalized dose, which causes the lines to overlap. For completeness, a shift to higher normalized doses (typically 10\% of the vertical scale of the graph) for combinations of low $r$ and high $s$ was observed, and these lines have been excluded from the graph for clarity. Furthermore, some combinations were not simulated due to the extremely long simulation time required, i.e., the combinations of high $r$ with low $s$ values. A strong dependence of the normalized saturation dose on the recombination probability can be observed from the figure. The very weak dependence of the normalized saturation dose on $s$ suggests the existence of a new regime that can be distinguished from the reaction- and the diffusion-limited regimes discussed earlier. In this new regime, the saturation is not limited by the reaction probability or diffusion rate but by the recombination loss. For the remainder of this paper, we refer to this new regime as the recombination-limited regime. Regarding the absolute saturation dose (instead of the normalized dose), the dose is similar to the $r$ $=0$ case in the near planar case $(\mathrm{AR}=1)$. The trends provided similar information as the normalized dose trends, and these absolute saturation dose trends are, therefore, not plotted.

By comparing loss probabilities from Table II with the trends in Fig. 4, consequences for possible ALD processes can be predicted. When using hydrogen radicals for the deposition of a material such as titanium, the recombination loss probability is high $(r=0.35)$ and achieving conformality will be difficult. However, for copper, which has a somewhat lower recombination probability for hydrogen radicals $(r=0.14)$, conformal deposition of trenches with AR $=9$ is more feasible and has indeed been reported. ${ }^{26}$ According to Fig. 4 in this case, conformal film growth requires a dose of $\sim 10$ times the dose required for planar surfaces. This dose apparently agrees with the dose used by the author: a $3 \mathrm{~s}$ plasma exposure was used, whereas in a previous publication it was stated that saturation occurred in less than $1 \mathrm{~s}$ for planar samples. ${ }^{32}$ Materials such as platinum have a lower recombination loss for hydrogen and it should already be much easier to deposit these materials in high AR structures by ALD processes employing hydrogen radicals. The 


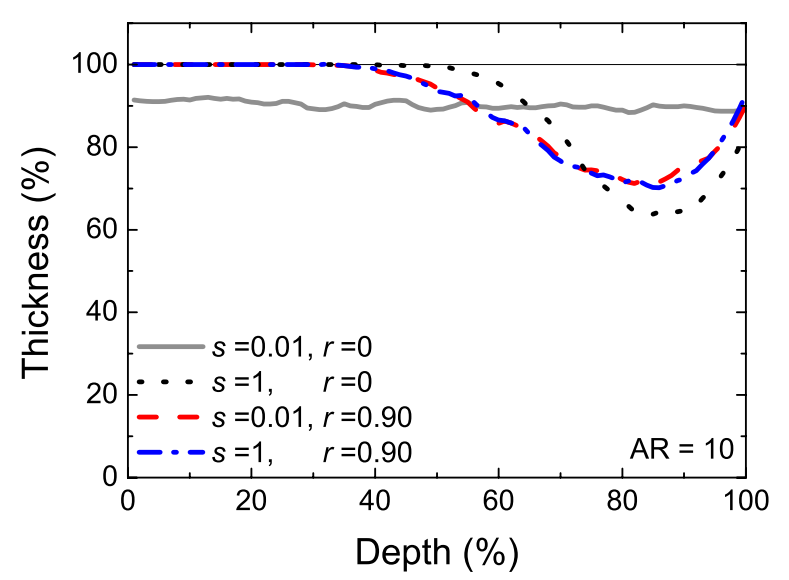

Figure 5. (Color online) Thickness as a function of depth for a trench of AR of 10 . The simulations were stopped at $90 \%$ coverage for the combinations of two reaction probabilities $(s=0.01$ and $s=1)$ with two loss probabilities $(r=0$ and $r=0.90)$.

same holds for metal oxide deposition by plasma-assisted ALD because the recombination probability of hydrogen, oxygen, and nitrogen radicals on oxides is low (Table II).

Figure 5 shows the effect of surface recombination loss on the deposition profile. The almost saturated profiles ( $90 \%$ coverage) obtained using low and high $s$ values are compared for $r=0$ and 0.90 . The cases of low and high $s$ with $r=0$ correspond well with the profiles expected for the reaction- and diffusion-limited cases, respectively. Remarkably, these two very distinct profiles converge to a single new profile when recombination is introduced with a large value of $r$. The value of $s$ in the case of high $r$ does not affect the profile. The new profile with recombination loss is similar to the profile for the diffusion-limited case in the sense that the profile has a minimum near the trench bottom. The shape of the profile is, however, different. How the value of $r$ affects the profile follows from a comparison of the deposition profiles at $90 \%$ saturation for a range of $r$ values (Fig. 6). The value of $s$ is chosen to be low enough $(s=0.001$ and $s=0.01)$ to have no observable effect on the profiles for the various $r$ values. Although all profiles are limited by the surface recombination loss process, no specific individual profile emerges. For higher $r$ values, the minimum near the bottom is deeper. Also, higher $r$ values require a much higher dose to achieve

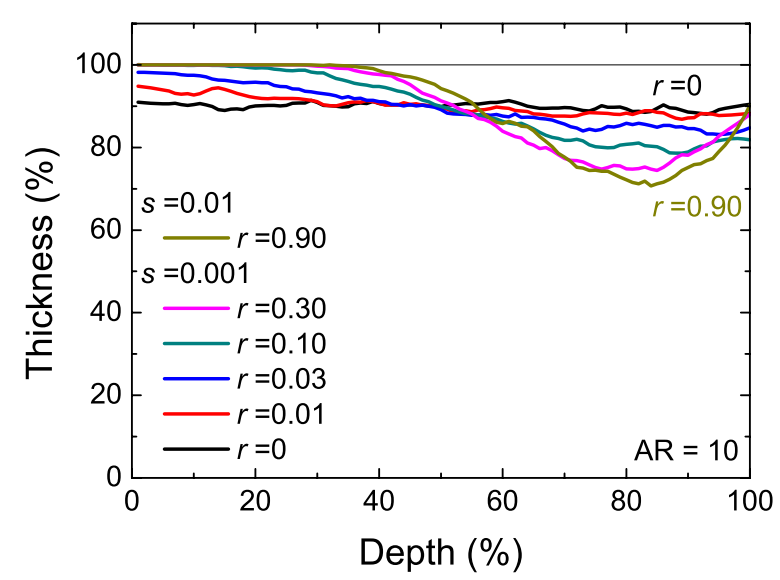

Figure 6. (Color online) Thickness as a function of depth for a trench with AR of 10. Simulations were stopped at $90 \%$ coverage for loss probabilities ranging from $r=0$ to $r=0.90$ at a low reaction probability $s=0.001$. (For $r=0.90$, the reaction probability value of $s=0.01$ was used to reduce the duration of the simulation.) the same coverage, as was shown in Fig. 4. Dendooven et al. stated that the recombination probability only affects the penetration depth but not the slope of the deposition profile. ${ }^{30}$ This is indeed the case for the conditions they investigated $(s=1$ and low $\mathrm{AR}=\sim 5)$. However, for lower $s$ values or higher values of AR, the shape of the deposition profile is affected by the value of $r$, as can be observed in Fig. 5 and 6.

Particle diffusion. - To study the diffusion of particles inside the trench for the three deposition regimes, the number of sidewall collisions as a function of position within the trench is compared for the three regimes. Figure 7 shows the wall collisions profiles for the three cases, which are assumed representative for the reaction-, diffusion-, and recombination-limited regimes. The number of collisions is expressed as collisions per percentage of depth, normalized to the total number of particles needed for saturation. These wall collisions profiles can be interpreted as the number of collisions along the trench wall in a specific time interval of the total time needed to reach saturation. The following observations can be made for the three regimes. Figure $7 \mathrm{a}$ shows that in the case of the reaction-limited regime, a flat profile of wall collisions is observed, which does not change during deposition. In this case, the loss of particles by reaction is low compared to the supply of particles by diffusion, and therefore, the particles can equilibrate throughout the trench by colliding with the bottom and coming back toward the top. Saturation occurs with an equal rate throughout the trench.

Figure $7 \mathrm{~b}$ shows the diffusion-limited case. Initially the number of wall collisions decreases quickly when going deeper inside the trench. The wall collisions profile changes during the deposition. The particle distribution extends further and further down into the trench with the progression of time. At the front of this particle distribution, the surface intervals are reacting, which stops the loss at that point. As a consequence, the particle diffusion extends further into the trench, which in effect causes a moving deposition front toward the bottom of the trench.

For the newly introduced recombination-limited regime (Fig. 7c), the wall collisions profile shows a strong decrease with depth, but different from the case in Fig. 7b, the wall collisions profile does not change when time progresses. Here, the decrease in the number of wall collisions with depth is not caused by the ALD reactions at the deposition front, as is the case for the diffusion-limited regime, but by the surface recombination loss. The more collisions a particle makes, the higher the chance of the particle being lost. The persistence of the profile is explained by the fact that the recombination loss process is also present on surface intervals where deposition has already taken place. Saturation can still occur throughout the trench but the rate of saturation is proportional to the wall collisions profile. The recombination loss processes are simulated in a very general way and only a constant loss probability is taken into account. The loss probability is likely to depend on the surface state and would therefore change with saturation, in turn causing an evolution of the wall collisions profile for the recombination-limited regime during deposition.

The deposition regimes. - The characteristics of the three deposition regimes can be described on the basis of the observations from the saturation doses and the deposition and the wall collisions profiles. The regimes are schematically depicted in Fig. 8, where the thickness can be interpreted either as the profile resulting from unsaturated growth or as the evolution of the saturation in the trench during the half-cycle. The first two depicted regimes have been suggested in the literature before; ${ }^{1}$ the recombination-limited regime is new and has not been identified previously to our knowledge. The regimes can be described as follows:

1. The reaction-limited regime (Fig. 8a): In this case, growth occurs simultaneously everywhere throughout the trench until saturation is reached. Almost no increase in dose is required with increasing $\mathrm{AR}$ as long as $s \ll H$. During the deposition, a constant particle density is present within the complete trench. 

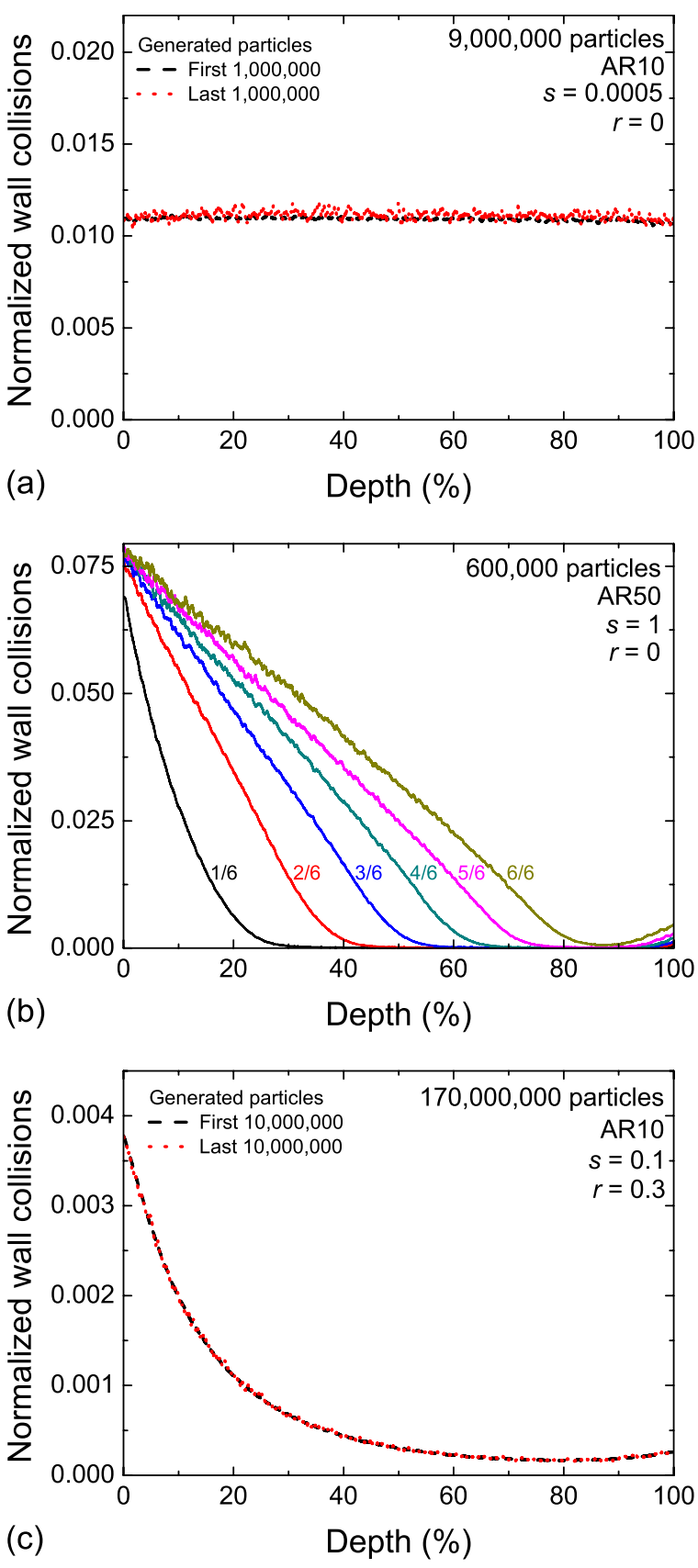

Figure 7. (Color online) Normalized number of wall collisions per percentage of depth within the trench during: (a) the first and last $1 \times 10^{6}$ particles of the reaction-limited deposition for $\mathrm{AR}=10$ and $s=0.0005$; (b) the 600,000 particles of the diffusion-limited deposition, sampled in steps of 100,000 particles (i.e., one-sixth to six-sixth) for $\mathrm{AR}=50$ and $s=1$; (c) the first and last $1 \times 10^{7}$ particles of the recombination-limited deposition for $\mathrm{AR}=10, s=0.1$, and $r=0.3$.

2. The diffusion-limited regime (Fig. 8b): In this case, a moving deposition front is observed. An increase in AR leads to a strong increase in saturation dose, whereas a change in $s$ has very little effect as long as $s \gg H$. The particle density in the trench decreases to zero at the deposition front.

3. The recombination-limited regime (Fig. 8c): In this case, growth occurs throughout the trench but the rate is reduced near the bottom of the trench (depending on $r$ ). At this position, saturation is reached the last. The saturation dose depends strongly on $r$ and also the shape of the deposition profile changes with $r$. Particles can be

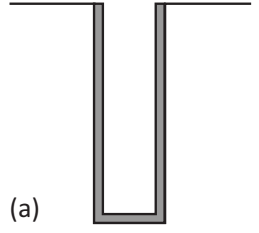

reaction-limited

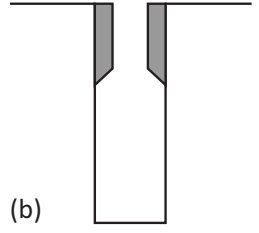

diffusion-limited

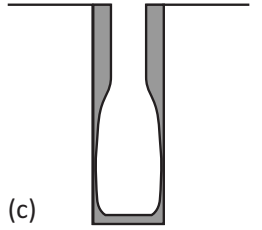

recombination-limited
Figure 8. Illustration of the three deposition regimes: (a) reaction-limited regime in which growth occurs simultaneously everywhere until saturation is reached; (b) diffusion-limited regime in which a downward moving saturation front is observed; and (c) recombination-limited regime in which growth occurs everywhere but with a rate that is reduced near the bottom of the trench where the condition of saturation is fulfilled the last. In (b), the small amount of growth at the bottom due to particles making very few collisions has been neglected.

present throughout the trench but their density decreases significantly toward the bottom.

Although the recombination-limited regime has not been identified as such in the literature, most depositions by plasma-assisted ALD in high AR structures are expected to occur in this regime. Kim et al. reported a not fully saturated deposition profile for $\mathrm{Ta}$ deposition by plasma-assisted ALD using a $\mathrm{H}_{2}$ plasma, which corresponds well with the observations for the not fully saturated deposition profiles obtained in the recombination-limited regime: ${ }^{28} \mathrm{~A}$ trench with $\mathrm{AR}=40$ was coated by Ta with the top part having $100 \%$ conformality and the lower part having $40 \%$ conformality. Most likely, longer plasma exposure times would be required to reach a conformal film inside the whole trench.

Transition between regimes. - As discussed earlier in the case of $r=0$, the process is either diffusion- or reaction-limited depending on $s$ and $\mathrm{AR}$, with $s=H$ as the transition condition between the regimes. A similar analytical relation would be useful to establish the location of the transition between the recombination-limited regime and the diffusion- and reaction-limited regimes. Walraven and Silvera reported an analytical description of the dissociation degree of hydrogen radicals leaving a tube in which surface recombination takes place. ${ }^{33}$ Using their relations, we have derived Eq. 1 (see Appendix), expressing the fraction of the particles $(\alpha)$ that reach the end of a tube or pore without recombining as a function of AR and surface recombination probability $r$

$$
\alpha=\frac{1}{\cosh (A R \sqrt{r})}
$$

For $\alpha=1$, none of the particles reaching the end of the pore have recombined, and for $\alpha=0$, all of the particles have recombined. Equation 1 shows that for low $\mathrm{AR}$ and $r, \alpha$ is close to 1 , whereas for high $\mathrm{AR}$ and $r, \alpha$ goes to zero. We define $\alpha=0.5$ as a transition condition between the recombination-limited regime and the other regimes. Figure 9 shows the resulting diagram for the three deposition regimes (reaction-, diffusion-, and recombination-limited) in the parameter space of recombination loss probability $r$ and AR. The line $s=H$, indicated for $s=0.01$ (solid red line) and $s=0.1$ (solid dashed line), defines the transition between the diffusion- and reaction-limited regimes. The black solid line represents the AR at which $50 \%$ of the particles have recombined $(\alpha=0.5)$ and defines the transition condition with the recombination-limited regime. In general, the situation is reaction-limited for low $r$ combined with a low $s$ and AR, whereas the situation is diffusion-limited for low $r$ combined with high $s$ and medium AR. The situation is recombination-limited for cases with low $r$ combined with high AR or, for all cases, with high $r$. These transition conditions are derived for pores instead of the trenches; however, the diagram and its transition conditions agree well with the results from our 2D trench 


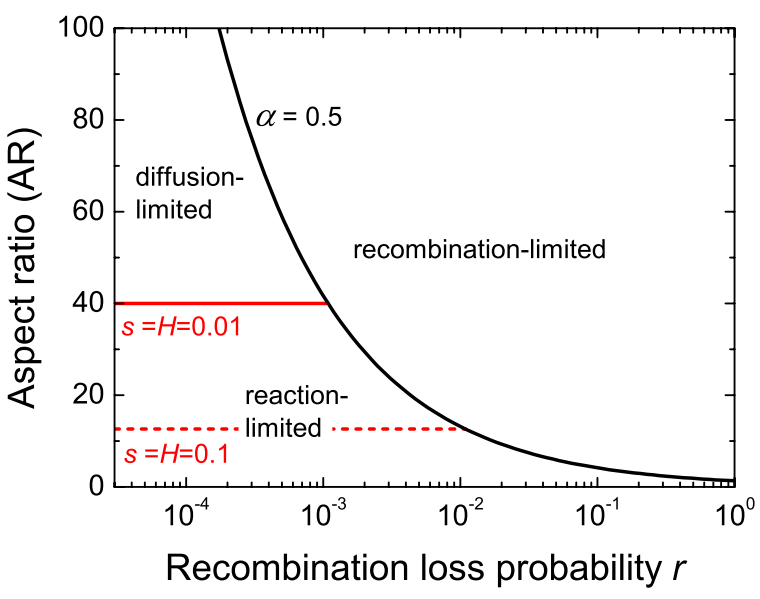

Figure 9. (Color online) Diagram showing the three deposition regimes represented in the parameter space of surface recombination loss probability $r$ and AR. The line $s=H$ for $s=0.01$ (solid red line) and $s=0.1$ (dashed red line) indicates the transition between the diffusion- and reaction-limited regimes. The black solid line represents the transition condition with the recombination-limited regime. This condition has been defined as the AR at which half of the particles have recombined $(\alpha=0.5)$ as a function of the recombination probability $r$.

simulations. Furthermore, the diagram clarifies the regime transition behavior. For instance, the value of $s$ has no effect on the condition whether a process is recombination-limited or not. The value of $s$ does, however, affect how the recombination-limited regime is entered. When moving down a trench, a process can either change from reaction-limited to diffusion-limited to recombination-limited (e.g., moving upward in Fig. 9 for $s=0.1$ at $r=2 \times 10^{-3}$ ) or the process can go directly from reaction-limited to recombinationlimited (e.g., moving upward in Fig. 9 for $s=0.01$ at $r=2$ $\left.\times 10^{-3}\right)$.

Implications.- Although due to the simplifications the applicability of the simulations might not be directly quantitative, the results obtained from the simulations certainly yield important qualitative information. From the simulations, it has been derived that deposition regimes with distinct behavior exist, which provides valuable insight in the ALD process in $3 \mathrm{D}$ structures. Although the simulations have been done in a $2 \mathrm{D}$ trenchlike geometry, the simulations also provide an insight into deposition in 3D structures. For instance, the analytical transition relations derived for pores hold fairly well for the simulated trench structures. Pores require longer precursor exposure for the same AR compared to trenches and, as a rule of thumb, can be considered equivalent to trenches of double $\mathrm{AR}^{5}$

When speculating on achievable ARs for coating 3D structures, the simulations suggest that for the plasma-assisted ALD processes with low recombination loss, ARs of 30 should be relatively straightforward. That is, compared to the dose required to saturate a planar surface, a relatively small increase in exposure is needed to saturate the 3D structure (factor of 4 for $r=0.01$ and $\mathrm{AR}=30$ ). For the high recombination loss processes, however, ARs higher than 10 might be impractical (the saturation dose increases by a factor of 41 for $r=0.30$ and $\mathrm{AR}=15$ ). In addition to the fact that plasmaassisted ALD is a relatively young field of research, this difficulty could be another reason for the small number of papers on conformal growth by plasma-assisted ALD in AR > 10 structures. Possible improvements in plasma-assisted ALD equipment (achieving much higher radical densities) would enable much higher ARs (e.g., $A R=100)$ assuming that additional effects such as possible damage by ion bombardment and film etching are not introduced in such a reactor design.
Ozone-based ALD processes. - Although we have focused on plasma-assisted ALD in this paper, the results of the simulations presented can also be applied to other ALD processes in which the reactant can undergo loss processes in addition to the ALD surface reactions. In principle, any reactant that can decompose or recombine at a surface without leaving a residue can be considered, for instance, ozone, formaldehyde, ${ }^{34}$ and hydrogen peroxide. ${ }^{35}$ Of these reactants, ozone $\left(\mathrm{O}_{3}\right)$ is most often employed and the surface loss process is reported to mainly consist of $\mathrm{O}_{3}$ recombination with surface $\mathrm{O}$ forming $\mathrm{O}_{2} \cdot{ }^{36,37}$ Ozone typically shows a very low loss probability at room temperature (on alumina $r=5 \times 10^{-6} 38$ and on stainless steel $r=3 \times 10^{-639}$ ), but is known to have higher decomposition rates at elevated temperatures. Moreover, ozone has particularly high decomposition rates on catalytic materials such as $\mathrm{MnO}_{2}$ and $\mathrm{Rh}_{2} \mathrm{O}_{3}{ }^{40,41}$ This means that achieving conformality for ozonebased processes of most materials should be relatively easy due to the low loss probability; however, the effects of loss are not negligible for very high ARs and for certain classes of materials. Further understanding of the dependence of the surface recombination probability on process parameters and on the chemical environment might provide ways to decrease the recombination loss and thereby improve the conformality. For instance, a low deposition temperature could be needed to coat a certain 3D structure when the recombination probability would be lower at reduced temperatures. To this end, the simulations described in this paper can be used to predict the saturation dose changes and the optimum deposition conditions.

\section{Conclusion}

The physical processes in plasma-assisted ALD that affect conformality were identified to be recombination loss of radicals, reaction probability of radicals, and particle diffusion of plasma species in the trench. The processes were generalized in terms of a general reaction probability $s$ and general loss probability $r$. A Monte Carlo model was developed to simulate the conformality of plasmaassisted ALD in a trench. For $r=0$, simulations in which the reaction probability and AR were varied led to the identification of a reaction- and a diffusion-limited regime. In the reaction-limited regime, the dose required to reach saturation depends on $s$ but not on the $\mathrm{AR}$ of the trench. Moreover, the deposition profiles before reaching saturation were flat. In the diffusion-limited regime, the dose increased strongly with AR, whereas the value of $s$ had little effect on the saturation dose. The value of $s$ does, however, affect the deposition profile because a lower $s$ results in a decrease in the slope of the thickness profile at the deposition front.

With the introduction of surface recombination loss in the simulations $(r>0)$, the saturation dose depended strongly on the value of the loss probability. Before reaching saturation, the deposition profiles of the wall of the trench showed a minimum near the bottom of the trench and the relative depth of this minimum increased with the loss probability. By combining these observations with those of the depth profiles of the wall collisions during deposition, three deposition regimes could be identified, namely a reaction-, a diffusion-, and a recombination-limited regime. The newly introduced recombination-limited regime is classified by a strong dependence of the saturation dose on the recombination loss probability $r$. The value of $r$ also affects the shape of the deposition profile causing a lower thickness of the film at the region in the trench toward the bottom. Analytical relations have been used to demarcate the regimes by defining transition conditions. The transition to the recombination-limited regime only depends on $r$ and AR.

Knowledge of the effective regime in which a deposition takes place yields the ability to predict the effect on conformality of a change in reactant type, reactant exposure, or AR. For plasmaassisted ALD in 3D structures, the process is usually limited by the surface recombination loss probability. Conformal deposition in high AR structures can, therefore, be achieved in the case of either a low loss probability or when a very large radical dose is applied. For low recombination loss, ARs of 30 should be relatively easy. For high recombination loss processes, an AR higher than 10 might be 
impractical. A further understanding of the dependence of the surface recombination probability on process parameters and on the chemical environment might provide ways of decreasing the recombination loss and thereby improve the conformality of plasmaassisted ALD films in 3D structures.

\section{Acknowledgments}

A. Versteegh and E. de Caluwé are thanked for their contribution to the development of the simulation software. This work was sponsored by the Materials Innovation Institute M2i (www.m2i.nl) under project no. MC3.06278 and by the Netherlands Technology Foundation STW.

\section{Appendix}

Equation A-1 shows our derived equation for the fraction of the particles $(\alpha)$ that reach the end of a tube or pore without recombining, as a function of AR and surface recombination probability $r$

$$
\alpha=\frac{1}{\cosh (A R \sqrt{r})}
$$

For $\alpha=1$, none of the particles reaching the end of the pore have recombined, and for $\alpha=0$, all of the particles have recombined. This equation was derived from the work of Walraven and Silvera, who studied the transmission of hydrogen radicals through a long tube (high AR) in the molecular flow regime and reported several analytical relations regarding the number of collisions and degree of dissociation of gas exiting the tube. ${ }^{33}$ To obtain Eq. A-1, the following steps have to be taken: The average number of collisions $N_{\mathrm{c}}$ that a particle makes after traveling a pore of length $l$ is given by Eq. 8 in the work by Walraven and Silvera and is rewritten to use $\mathrm{AR}^{33}$

$$
N_{\mathrm{c}}=\frac{1}{8}\left(\frac{l}{b}\right)^{2}=\frac{1}{2} \mathrm{AR}^{2}
$$

where $b$ represents the radius and $\mathrm{AR}=l / 2 b$. This equation is inserted in Eq. $9 \mathrm{~b}$ from Walraven and Silvera, resulting in Eq. A-3 below, which after simplification results in Eq. A-1 used in this work

$$
\alpha=\frac{1}{\cosh \left(\sqrt{2 r N_{\mathrm{c}}}\right)}=\frac{1}{\cosh (A R \sqrt{r})}
$$

Eindhoven University of Technology assisted in meeting the publication costs of this article.

\section{References}

1. J. W. Elam, D. Routkevitch, P. P. Mardilovich, and S. M. George, Chem. Mater, 15, 3507 (2003)

2. R. G. Gordon, D. Hausmann, E. Kim, and J. Shepard, Chem. Vap. Deposition, 9, 73 (2003).

3. B. Dhandapani and S. T. Oyama, Appl. Catal., B, 11, 129 (1997).

4. A. Burke, G. Braeckelmann, D. Manger, E. Eisenbraun, A. E. Kaloyeros, J. P. Mcvittie, J. Han, D. Bang, J. F. Loan, and J. J. Sullivan, J. Appl. Phys., 82, 4651 (1997).

5. H. C. Wulu, K. C. Saraswat, and J. P. Mcvittie, J. Electrochem. Soc., 138, 1831 (1991).

6. J. Dendooven, D. Deduytsche, J. Musschoot, R. L. Vanmeirhaeghe, and C. Detavernier, J. Electrochem. Soc., 156, P63 (2009).

7. J. Y. Kim, J. H. Ahn, S. W. Kang, and J. H. Kim, J. Appl. Phys., 101, 073502 (2007).
8. A. M. Lankhorst, B. D. Paarhuis, H. J. C. M. Terhorst, P. J. P. M. Simons, and C. R. Kleijn, Surf. Coat. Technol., 201, 8842 (2007).

9. G. Prechtl, A. Kersch, G. Schulze Icking-Konert, W. Jacobs, T. Hecht, H. Boubekeur, and U. Schröder, Tech. Dig. - Int. Electron Devices Meet., 2003, 245.

10. M. Rose and J. W. Bartha, Appl. Surf. Sci., 255, 6620 (2009).

11. S. B. S. Heil, J. L. Hemmen, C. J. Hodson, N. Singh, J. H. Klootwijk, F. Roozeboom, M. C. M. van de Sanden, and W. M. M. Kessels, J. Vac. Sci. Technol. A, 25, 1357 (2007)

12. H. B. Profijt, P. Kudlacek, M. C. M. van de Sanden, and W. M. M. Kessels, J. Electrochem. Soc., To be published.

13. R. L. Puurunen, J. Appl. Phys., 97, 121301 (2005)

14. F. Greer, D. Fraser, J. W. Coburn, and D. B. Graves, J. Vac. Sci. Technol. A, 21, 96 (2003).

15. M. Rose, J. W. Bartha, and I. Endler, Appl. Surf. Sci., 256, 3778 (2010).

16. J. Y. Kim, J. H. Kim, J. H. Ahn, P. K. Park, and S. W. Kang, J. Electrochem. Soc., 154, H1008 (2007).

17. E. N. Denbnovetskaya, V. A. Lavrenko, I. A. Podchernyaeva, T. G. Protsenko, N. I Siman, and V. S. Fomenko, Powder Metall. Met. Ceram., 10, 289 (1971).

18. J. C. Greaves and J. W. Linnett, Trans. Faraday Soc., 55, 1346 (1959).

19. J. C. Greaves and J. W. Linnett, Trans. Faraday Soc., 55, 1355 (1959).

20. B. J. Wood and H. Wise, J. Phys. Chem., 65, 1976 (1961).

21. J. L. van Hemmen, S. B. S. Heil, J. H. Klootwijk, F. Roozeboom, C. J. Hodson, M. C. M. van de Sanden, and W. M. M. Kessels, J. Electrochem. Soc., 154, G165 (2007).

22. N. G. Kubala, P. C. Rowlette, and C. A. Wolden, J. Phys. Chem. C, 113, 16307 (2009).

23. A. Niskanen, U. Kreissig, M. Leskela, and M. Ritala, Chem. Mater, 19, 2316 (2007).

24. J. Y. Kim, D. Y. Kim, H. O. Park, and H. T. Jeon, J. Electrochem. Soc, 152, G29 (2005).

25. J. S. Park, H. S. Park, and S. W. Kang, J. Electrochem. Soc., 149, C28 (2002).

26. L. Wu and E. Eisenbraun, J. Electrochem. Soc., 156, H734 (2009).

27. A. Niskanen, T. Hatanpaa, K. Arstila, M. Leskela, and M. Ritala, Chem. Vap. Deposition, 13, 408 (2007).

28. H. Kim, C. Cabral, C. Lavoie, and S. M. Rossnagel, J. Vac. Sci. Technol. B, 20, 1321 (2002).

29. W. M. M. Kessels, Baltic Conference on Atomic Layer Deposition 2006, Centre for Materials Science and Nanotechnology, p. 20 (2006).

30. J. Dendooven, D. Deduytsche, J. Musschoot, R. L. Vanmeirhaeghe, and C. Detavernier, J. Electrochem. Soc., 157, G111 (2010).

31. I. Perez, E. Robertson, P. Banerjee, L. Henn-Lecordier, S. J. Son, S. B. Lee, and G W. Rubloff, Small, 4, 1223 (2008).

32. L. Wu and E. Eisenbraun, J. Vac. Sci. Technol. B, 25, 2581 (2007).

33. J. T. M. Walraven and I. F. Silvera, Rev. Sci. Instrum., 53, 1167 (1982).

34. Y. Sekine, Atmos. Environ., 36, 5543 (2002).

35. M. Pradhan, M. Kalberer, P. T. Griffiths, C. F. Braban, F. D. Pope, R. A. Cox, and R. M. Lambert, Environ. Sci. Technol., 44, 1360 (2010).

36. W. Li, G. V. Gibbs, and S. T. Oyama, J. Am. Chem. Soc., 120, 9041 (1998)

37. W. Li and S. T. Oyama, J. Am. Chem. Soc., 120, 9047 (1998).

38. R. C. Sullivan, T. Thornberry, and J. P. D. Abbatt, Atmos. Chem. Phys., 4, 1301 (2004).

39. H. Itoh, I. M. Rusinov, S. Suzuki, and T. Suzuki, Plasma Processes Polym., 2, 227 (2005)

40. S. T. Oyama, Catal. Rev - Sci. Eng., 42, 279 (2000).

41. J. Hämäläinen, F. Munnik, M. Ritala, and M. Leskelä, J. Electrochem. Soc., 156, D418 (2009).

42. Y. C. Kim and M. Boudart, Langmuir, 7, 2999 (1991)

43. A. D. Tserepi and T. A. Miller, J. Appl. Phys., 75, 7231 (1994).

44. J. Abrefah and D. R. Olander, Surf. Sci., 209, 291 (1989).

45. A. Bouchoule and P. Ranson, J. Vac. Sci. Technol. A, 9, 317 (1991).

46. S. F. Adams and T. A. Miller, Plasma Sources Sci. Technol, 9, 248 (2000).

47. M. Mozetic and A. Zalar, Appl. Surf. Sci., 158, 263 (2000). 